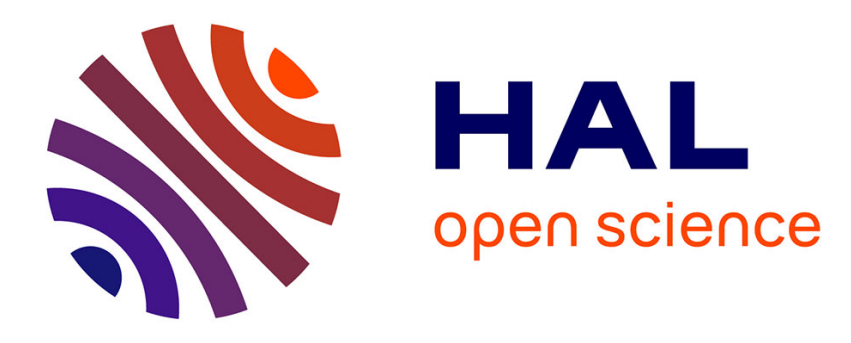

\title{
Robustness against unknown Networked induced Delays of Observer based FDI
}

Dominique Sauter, Taha Boukhobza

\section{To cite this version:}

Dominique Sauter, Taha Boukhobza. Robustness against unknown Networked induced Delays of Observer based FDI. Aug 2006, pp.CDROM. hal-00120894

\section{HAL Id: hal-00120894 \\ https://hal.science/hal-00120894}

Submitted on 18 Dec 2006

HAL is a multi-disciplinary open access archive for the deposit and dissemination of scientific research documents, whether they are published or not. The documents may come from teaching and research institutions in France or abroad, or from public or private research centers.
L'archive ouverte pluridisciplinaire HAL, est destinée au dépôt et à la diffusion de documents scientifiques de niveau recherche, publiés ou non, émanant des établissements d'enseignement et de recherche français ou étrangers, des laboratoires publics ou privés. 


\title{
Robustness against unknown Networked induced Delays of Observer based FDI
}

\author{
Dominique Sauter and Taha Boukhobza \\ Centre de Recherche en Automatique de Nancy \\ University Henri Poincaré, Nancy- CNRS UMR 7039 \\ BP 239 - 54506 Vandoeuvre Cedex - France. \\ Email:_dominique.sauter@cran.uhp-nancy.fr
}

\begin{abstract}
In this paper, we are concerned by the problem of Fault diagnosis in Networked Control Systems (NCS). The effect of unknown networked induced delays on conventional observed based residual generator is studied. It is shown that the detection performances may be reduced due to the sensitivity of the residuals to the delays. With the assumption that the network delays belong to a given bounded set, in order to enhance the robustness of fault detection an adaptive evaluation procedure of the residuals is proposed. The detection thresholds which depend on the maximum influence of the network delays are estimated using an optimization approach.
\end{abstract}

Keywords: Fault detection, observer based residual generation, networked control systems, unknown delays, robustness.

\section{INTRODUCTION}

The new trends for the realization of fault diagnosis (FDI) and fault tolerant control (FTC) systems that implement supervision functionalities (performance evaluation, fault diagnosis) and reconfiguration mechanisms are to implement them thanks to cooperative functions that are also distributed on a networked architecture. The study and the design of such application, called Networked Control Systems (NECS) [9], represent an emerging important research field.

Conventional theories with ideal assumptions, such as non-delayed sensing and actuation must be re-evaluated. Unknown network induced delays [5], [6].and data dropouts [3], [4] are the main characteristics of the network that are to be considered in the design of FDI However, whereas there are a great number of significant results for the control problems of NECS, studies related to FDI of NECS are just at the starting point.

In this paper we are interested on evaluating first the effects of the communication induced delays in the performance of the FDI system. It will be shown that the performances of FDI are altered by the delay. Depending on the input signal applied to the system, delays can even induce false alarms. As a solution to the robustness problem an adaptive thresholding mechanism is proposed for residual evaluation.

The proposed approach for threshold adaptation is based on the optimisation of a performance index related to the maximum amplitude of the residual with respect to the possible variation of the delays inside a bounded region.

Simulations are performed for the validation of the proposed method.

\section{INSTANTANEOUS EXECUTION OF FDI METHODS}

In this section, we introduce the traditional model-based FDI methods. Let us consider the system:

$$
\left\{\begin{array}{c}
\dot{x}(t)=A x(t)+\sum_{i=1}^{m} B_{i} u_{i}(t)+E f(t) \\
y(t)=C x(t)+F f(t)
\end{array}\right.
$$

where $x(t) \in \mathfrak{R}^{n}$ is the state vector, $u(t) \in \mathfrak{R}^{m}$ the input vector, $y(t) \in \mathfrak{R}^{p}$ the output vector and $f(t) \in \mathfrak{R}^{q}$ represents fault vectors to be 
identified. All the matrices in Eq. (1) are of appropriate dimensions.

Here, it is assumed the residual generation and evaluation algorithms are executed instantaneously at every sampling period $k T$.

Based on this assumption, if the control input is kept constant over each sampling interval $T$, and if we also consider that fault input is constant over this interval, the discrete time system can be described by:

$\left\{\begin{array}{l}x((k+1) T)=\Phi x(k T)+\sum_{i=1}^{m} \Gamma_{i} u_{i}(k T)+\Xi f(k T) \\ y(k T)=C x(k T)+F f(k T)\end{array}\right.$

where :

$\Phi=e^{A T}, \Gamma_{i}=\int_{0}^{T} e^{A s} B_{i} d s, \Xi=\int_{0}^{T} e^{A s} E d s$

The aim of failure diagnosis systems is to design a residual which is zero in the fault free case and non zero when a fault occurs. The problem to deal with is to design a linear filter whose inputs are the measurable outputs and control inputs which generates certain outputs called residual signals, one for each possible fault, not affected by other faults and not affected by disturbances.

In this paper, we consider a classical observer based residual generator. Therefore, our aim is to find an observer for system of Eq. (2) of the form:

$$
\left\{\begin{array}{c}
\hat{x}((k+1) T)=\Phi \hat{x}(k T)+\sum_{i=1}^{m} \Gamma_{i} u_{i}(k T) \\
+L(y(k T)-\hat{y}(k T)) \\
\hat{y}(k T)=C \hat{x}(k T)
\end{array}\right.
$$

and the residual generator :

$$
r(k T)=T(y(k T)-\hat{y}(k T))
$$

where $L \in \mathfrak{R}^{n, p}$ an $T \in \mathfrak{R}^{q, p}$ are matrices that are designed in order to fulfill fault detection and isolation requirements.

From (5) and (6), the estimation error $\varepsilon(k T)=x(k T)-\hat{x}(k T)$ and the output of the filter propagate as :

$$
\left\{\begin{array}{l}
\varepsilon((k+1) T)=(\Phi-L C) \varepsilon(k T)+(E-L F) f(k T) \\
r(k T)=T C \varepsilon(k T)
\end{array}\right.
$$

Actually, there are various approaches [2],[7] to determine the gain matrices $L$ and $T$, but we do not discuss this topic in the paper. The residual generator given by Eqs. (4) and (5) will be considered in the next section to study the effect of network induced delays.

\section{MODELLING OF NETWORK INDUCED DELAYS}

If, it is now supposed that the system is controlled over a network, then we have to take into account the sensor to controller $\tau_{s c}$ delays and controller to actuator delays $\tau_{c a}$ as it illustrated by Fig. 1.

Note that the delays, in general, cannot be considered constant and known. Network induced delays may vary, depending on the network traffic, medium access protocol and the hardware.

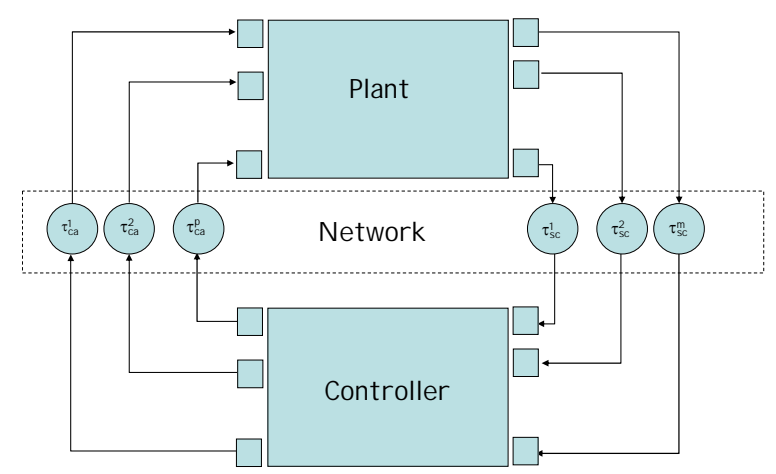

Fig 1: A feedback control system with network induced delays

From the controller viewpoint the system of eq (1) can be described by the model :

$$
\left\{\begin{array}{l}
\dot{x}(t)=A x(t)+\sum_{i=1}^{m} B_{i} u_{i}\left(t-\tau_{i}\right)+E f(t) \\
y(t)=C x(t)
\end{array}\right.
$$

where the delays $\tau_{i}$ depend on the sensor to controller and controller to actuator delays. This can be more clearly seen in Fig 2., in which $k T$ are the sensor sampling time and the values $y(k T)$ arrives at the controller after a delay of $\tau_{s c}$. When the controller receives the sensor value, it immediately computes the new control input value $u(k)$ and a ' $\mathrm{ZOH}$ ' device holds this value constant until the next sensor reading. The control signal is received by the actuator after a delay of $\tau_{c a}$.

The discrete time model of Eq (1) is now given by :

$$
\begin{aligned}
x((k+1) T)= & \Phi x(k T)+\sum_{i=0}^{m}\left(\Gamma_{0 i} u_{i}(k T)\right) \\
& +\sum_{i=0}^{m}\left(\Gamma_{l i} u_{i}((k-1) T)\right)+\Xi f(k T)
\end{aligned}
$$

with the definitions :

$$
\Gamma_{0 i}=\int_{0}^{T-\tau_{i}} e^{A s} B_{i} d s \quad \Gamma_{l i}=\int_{T-\tau_{i}}^{T} e^{A s} B_{i} d s
$$




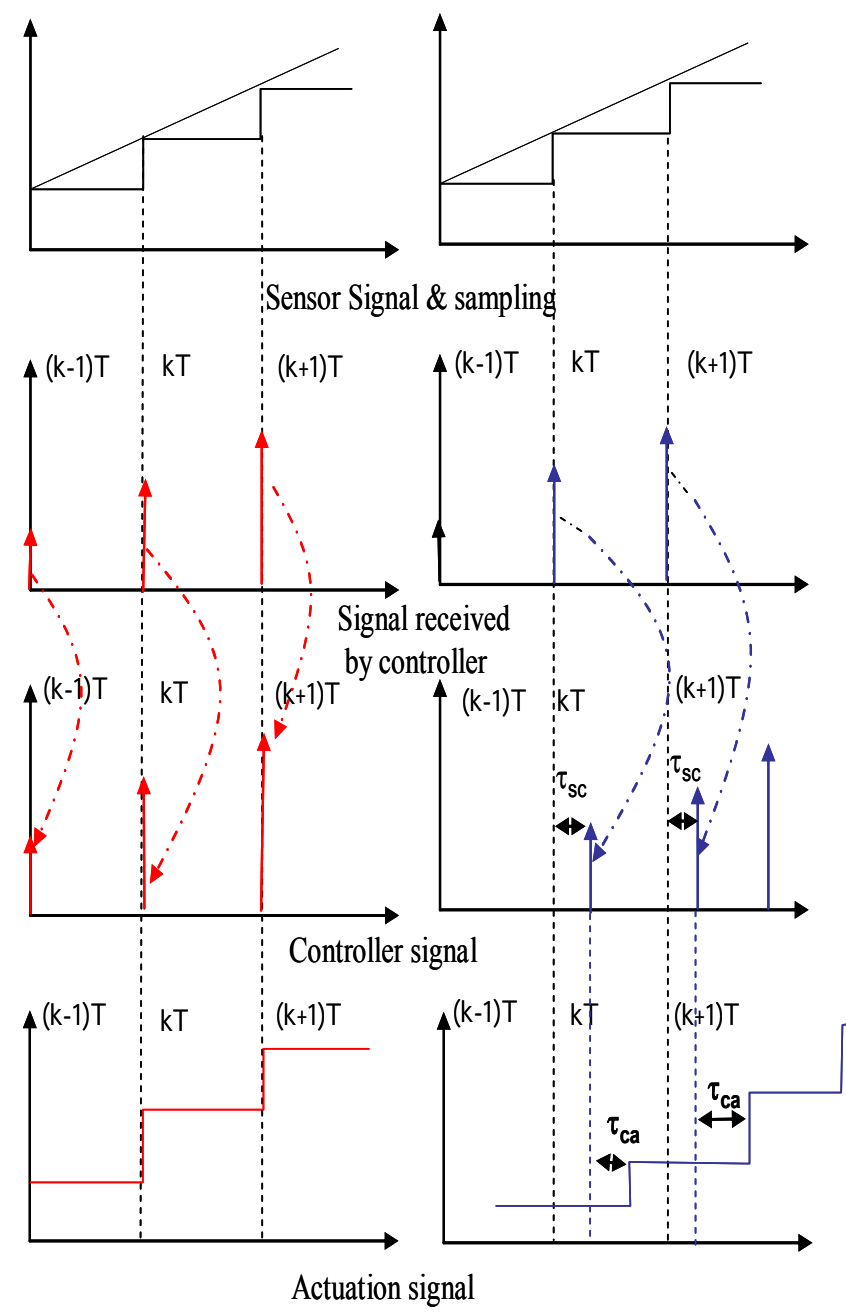

Fig. 2. : Delay timing diagram for a networked control system.

Eq. (8) can alsobe rewritten as :

$$
\left\{\begin{array}{c}
x((k+1) T)=\Phi x(k T)+\sum_{i=1}^{m}\left(\Gamma u_{i}(k T)\right) \\
-\sum_{i=1}^{m}\left(\Gamma_{l i} \Delta u_{i}(k T)\right)+\Xi f(k T) \\
y(k)=C x(k)+F f(k)
\end{array}\right.
$$

with :

$$
\left.\Delta u_{i}(k T)=u_{i}(k T)-u_{i}(k-1) T\right)
$$

The FDI system introduced in section 2 was designed a priori to achieve certain performances characteristic without considering the time delays.

Let us now examine the effects of network induced delays on to the residual generator given by $\mathrm{Eq}(5)$.

From Eqs. (10) and (5), the estimation error $e(k T)=x(k T)-\hat{x}(k T)$ and the residual vector $r(k T)$ propagate as :

$$
\left\{\begin{array}{l}
\varepsilon((k+1) T)=(\Phi-L C) \varepsilon(k T) \\
\quad+\sum_{i=1}^{m} \Gamma_{l i}\left(\tau_{i}\right) \Delta u_{i}(k T)+(\Xi-L F) f(k T) \\
r(k T)=T C e(k T)
\end{array}\right.
$$

Hence, it appears that the robustness of the fault diagnosis system against network induced delays depend on the amplitude of the unknown term $\sum_{i=1}^{m} \Gamma_{l i}\left(\tau_{i}\right) \Delta u_{i}(k T)$.

We consider that a fault is detectable if the evaluation function $\Psi(t)=|r(t)|$ given in the temporal domain exceeds pre-definite thresholds as follows :

$$
\begin{cases}\Psi\left(r_{i}(k T) / f_{j}\right) \geq T h(k T) & \text { for } f_{j} \neq 0 \\ \Psi\left(r_{i}(k T) / f_{j}\right)<T h(k T) & \text { for } f_{j}=0\end{cases}
$$

Thus, the detection thresholds $\operatorname{Th}\left(f_{j}\right)$ depends upon the maximum amplitude of the uncertainties induced by the network delays :

$\operatorname{Th}\left(r_{i}(k T) / f_{j}\right)=\underset{\tau}{\operatorname{Sup}}\left|r_{i}(k T)\right|=\underset{\tau}{\operatorname{Sup}}\left|T \varepsilon_{i}(k T)\right|$

If we assume that the delays are bounded :

$0 \leq \tau \leq \tau^{+}$, then with :

$\left|r^{+}(k T)\right|=\operatorname{Sup}_{0 \leq \tau \leq \tau^{+}}|r(k T)|$

the following inequalities hold :

$0 \leq|r(k T)| \leq\left|r^{+}(k T)\right|$

making thus possible to determine the optimal threshold for fault detection.

Several approaches have been proposed in the literature for adaptive thresholding [1] and interval analysis [8]. To determine the optimal threshold we suggest to consider an optimisation approach.

\section{Adaptive Residual eVAluation}

The aim is to determine the maximum amplitude of the residuals, considering the influence of the unknown network induced delays.

In the continuous time domain, over the sampling period $[k T,(k+1) T]$ the system model satisfy to :

$$
\left\{\begin{array}{c}
\dot{x}(t)=A x(t)+\sum_{i=1}^{m} B_{i} u_{i}\left(t, \tau_{i}\right)+E f(t) \\
y(t)=C x(t)+F f(t)
\end{array}\right.
$$

with the control input :

$$
\left\{\begin{array}{l}
u_{i}\left(t, \tau_{i}\right)=u_{i}(k T-1), \quad k T \leq t \leq k T+\tau_{i} \\
u_{i}\left(t, \tau_{i}\right)=u_{i}(k T), \quad k T+\tau_{i} \leq t \leq(k+1) T
\end{array}\right.
$$

Over the same sampling period, the discrete time observer given by equation (5) is, in the continuous time domain, equivalent to : 


$$
\left\{\begin{array}{l}
\dot{\dot{x}}(t)=A \hat{x}(t)+\sum_{i=1}^{m} B_{i} u_{i}(t)+L(y(t)-\hat{y}(t)) \\
\hat{y}(t)=C \hat{x}(t)
\end{array}\right.
$$

where $L$ is such as $\Phi-K C=\exp \{(A-L C) T\}$.

But, since the control input applied to the observer

is $u_{i}(t, 0)=u_{i}(k T), \quad k T \leq t \leq(k+1) T$

the estimation error $\varepsilon(t)=x(t)-\hat{x}(t)$ and

the residual propagate as

$$
\left\{\begin{array}{l}
\dot{\varepsilon}(t)=(A-L C) \varepsilon(t)+\sum_{i=1}^{m} B_{i}\left(u_{i}\left(t, \tau_{i}\right)-u_{i}(t, 0)\right) \\
r(t)=T C \varepsilon(t)
\end{array}\right.
$$

Note that in comparison to Eq.(12), we have $\left.-\Delta u_{i}(k T)=u_{i}\left(t, \tau_{i}\right)-u_{i}(t, 0)\right)$.

The minimum threshold for fault detection, can be considered as a performance index to be maximized for the dynamic system described by Eq (20). Thus, the optimisation problem is to find the inputs $u_{i}^{*}(t)$ on the time interval $[k T,(k+1) T]$ such as the performance index $\Psi(t=k T)=|r(k T)|$ is maximised.

For that purpose, let us introduce the Hamiltonian :

$$
\begin{aligned}
H=\lambda^{T} & {[(A-L C) \varepsilon(t)} \\
& \left.+\sum_{i=1}^{m} B_{i}\left(u_{i}\left(t, \tau_{i}\right)-u_{i}(t, 0)\right)\right]
\end{aligned}
$$

where $\lambda$ is the co-state vector.

The solution of the optimisation problem satisfy to the state and co-state equations :

$$
\begin{aligned}
\dot{\varepsilon}^{*}(t)=\frac{\partial H}{\partial \lambda^{*}}= & (A-L C) \varepsilon^{*}(t) \\
& +\sum_{i=1}^{n} b_{i}\left(u_{i}^{*}(t)-u_{i}(t, 0)\right) \\
\dot{\lambda}^{*}(t)=-\frac{\partial H}{\partial \varepsilon}= & -(A-L C) \lambda^{*}(t)
\end{aligned}
$$

where * stands for the optimum and $u_{i}^{*}(t)=u_{i}\left(t, \tau_{i}^{*}\right)$ is given by the application of the Pontryagin minimum principle:

$$
\tau_{i}^{*}=\underset{0 \leq \tau_{i} \leq \tau_{i}^{+}}{\operatorname{Arg}}(\operatorname{Min}(H))
$$

In addition to the initial conditions $\varepsilon(k T)$, the terminal boundary conditions for this fixed terminal time problem is :

$\left[\frac{\partial \Psi}{\partial \varepsilon}-\lambda(t)\right]_{t=(k+1) T}=0$

which give : $\lambda((k+1) T)=T C\left[\begin{array}{c}\operatorname{sgn}\left(\varepsilon_{1}(k+1) T\right) \\ \vdots \\ \operatorname{sgn}\left(\varepsilon_{n}(k+1) T\right)\end{array}\right]$

Thus, the optimisation consists of solving state and co-state equations with boundary conditions while selecting at all time those values equations $\tau_{i}^{*}$ that maximize $H$. The optimisation problem is very simple since over the length of optimisation window only one commutation of the control input is applied.

Therefore the solution to (21) is given by :

$$
\tau^{*}=\operatorname{Arg}\left(\operatorname{Min}\left(\lambda^{T} \sum_{i=1}^{m} B_{i} \Delta u_{i}\left(t, \tau_{i}\right)\right)\right)
$$

Obviously, the optimum for $\tau_{i}^{*}$ (either $\tau_{i}^{+}$or 0 ) depends only upon the sign of the terms in $\lambda^{T} \sum_{i=1}^{m} B_{i} u_{i}\left(t, \tau_{i}\right)$ which requires to know the sign of $\lambda(t)$.

This can be achieved when solving (23). Since the inputs over the considered time interval input, are of step type

The optimization procedure can be iterated over several sampling intervals.

\section{APPLICATION}

The process pictured in Fig.3. is considered for illustration purpose. The plant is composed of two tanks of section $S$ supplied by two pumps with flow rates $Q_{1}$ and $Q_{2}$. The two tanks are connected through a pipe. Output flow rate is delivered at the outlet of the second tank. Input control variable are the input flow-rates. Measurement output variables as well as state variables are the levels $L_{1}$ and $L_{2}$ in the two tanks.

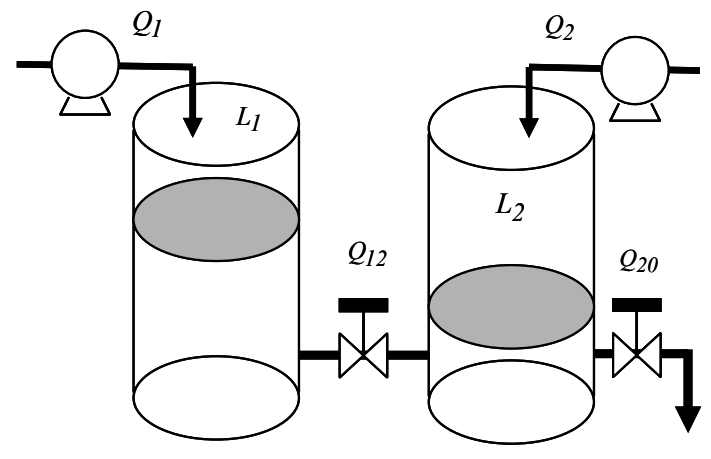

Fig. 3. Physical description of the Benchmark

As an approximation, with $L_{0}=0$ it is supposed that the out flow-rates depends on the tank levels as follows:

$Q_{i j}=R\left(L_{i}-L_{j}\right)$

The system which is a $2^{\text {nd }}$ order one with 2 inputs and 2 outputs can be modelized in 
the form of the state space representation (1) with the following matrices :

$$
\begin{aligned}
& A=\left[\begin{array}{cc}
\frac{-1}{R S} & \frac{1}{R S} \\
\frac{1}{R S} & \frac{-2}{R S}
\end{array}\right], \quad B=\left[\begin{array}{cc}
\frac{1}{S} & 0 \\
0 & \frac{1}{S}
\end{array}\right] \\
& C=\left[\begin{array}{ll}
1 & 0 \\
0 & 1
\end{array}\right], E=0, F=C .
\end{aligned}
$$

Only sensor faults have been considered in the simulations.

For residual generation, two state observers driven by a single output (either $L_{1}$ or $L_{2}$ were designed in order to given a estimation of the other outputs. This dedicated scheme is a classical one for sensor fault detection and isolation.

In the simulations which were performed, the sampling period is $1 \mathrm{~s}$ and the network induced delays are simulated as random variables constrained to belong to the interval $[0,1 s]$. Output signal \#1 is of a sin wave type, while input signal \#2 is of step type. The obtained results are reported on Fig.5 and Fig. 6. which show the results obtained with the 2 observers. Each figure gives respectively the output signal $\left(L_{1}\right.$ or $\left.L_{2}\right)$ with the additive fault signal, the dedicated residuals when there is no network delays $r_{1}=L_{1}-\hat{L}_{1}$ or $r_{2}=L_{2}-\hat{L}_{2}$, and the residuals influenced by the delays . The bottom plots show clearly using for residual evaluation the adaptive threshold developed in section 4 will enhance the robustness of the detector.

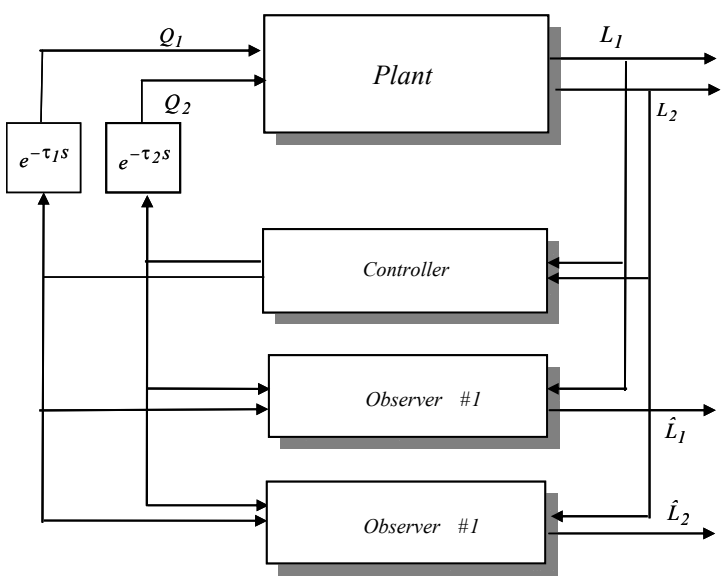

Fig. 4: Simulator for FDI of a Networked control system

\section{CONCLUSION}

This paper presents an adaptive residual evaluation strategy suitable for fault diagnosis of networked Control systems. The network induced delays are considered as unknown bounded variables. To determine the adaptive threshold an optimization procedure has been used.

Simulations resulys have shown the effectivness of the proposed method.
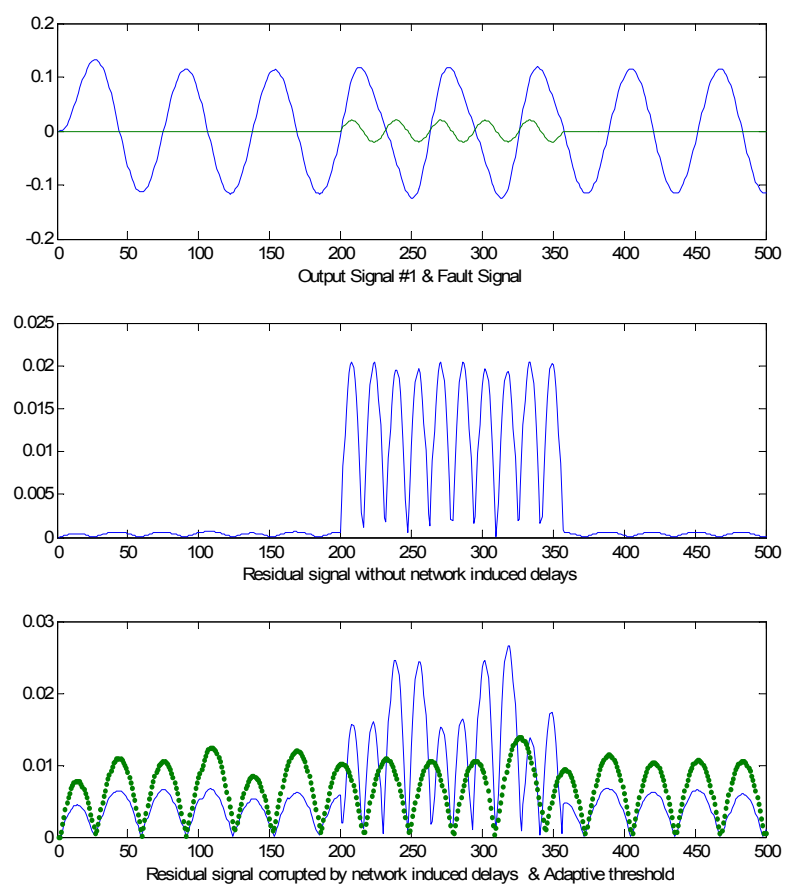

Fig 5. Simulation results obtained with observer \#1.
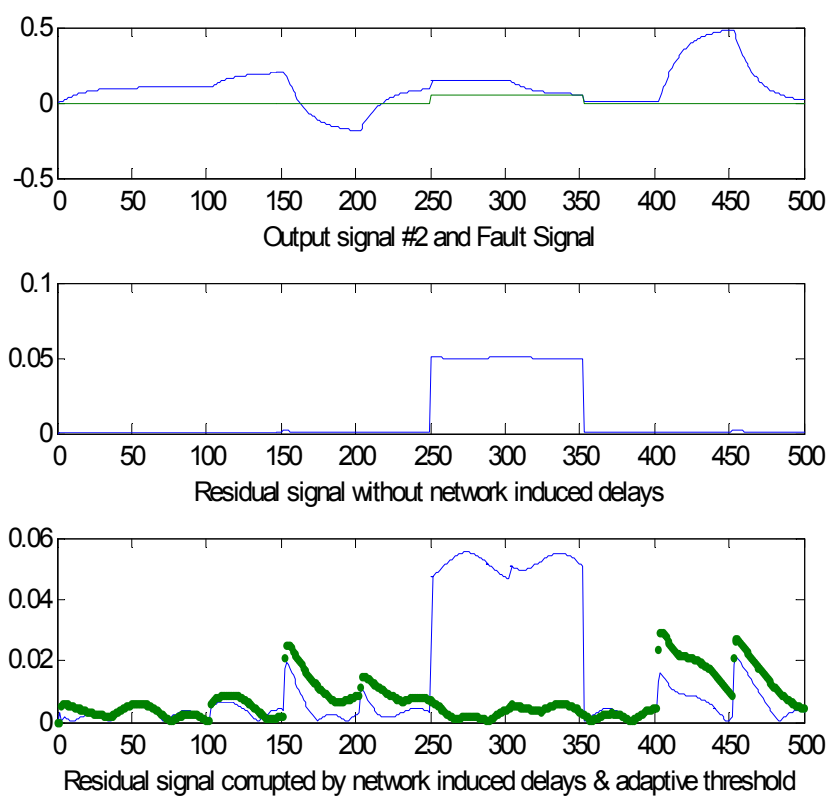

Fig 6. Simulation results obtained with observer \#2.

\section{ACKNOWLEDGEMENT}

Partial support from the European project NeCST. (NeCST EU-IST-2004-004303) is gratefully acknowledged.

\section{REFERENCES}


[1]Emami-Naeini A., Akhter M.M. and Rock S.M., "Effect of model uncertainty on failure detection: the threshold selector", IEEE Trans. Aut. Control 33, p. 1106-1115, 1988.

[2]Frank, P., \& Ding, S. Current development in the theory of FDI. Proc. IFAC Safeprocess (16-27), Budapest, Hungary., 2000.

[3] Ling Q. and Lemmon M.D. Robust performance of soft real-time networked control systems with data dropouts», Proc. Of the IEEE Conference on Decision and Control, 2002.

[4]Ling Q. and Lemmon M.D, «Soft real-time scheduling of networked systems with dropouts governed by a Markov chain», Proc. Of the American Conference on Control, 2003.

[5] Nilsson. JReal-Time Control Systems with Delays. PhD thesis, IT Lünd (Sweden), 1998.

[6] Nilsson J. et al, "Stochastic analysis and c6ontrol of real-time systems with random time delays", Automatica, vol.34, pp.57-64, 1998.

[7] Patton R.J., P. Frank, R. Clark (Ed.), Fault diagnosis in dynamic systems, Prentice Hall, International series in systems and control engineering., 1989.

[8] Puig V. , Quevedo J., Escobet T. , De las Heras S., Passive robust Fault detection approaches using interval models., 15th Triennial World Congress of the International Federation of Automatic Control Barcelona, 21-26 July 2002.

[9] W. Zhang, M. S. Branicky, and S. M. Philipps. Stability of networked control systems. IEEE Control Systems Magazine, 21(1):84-99, 2001 